
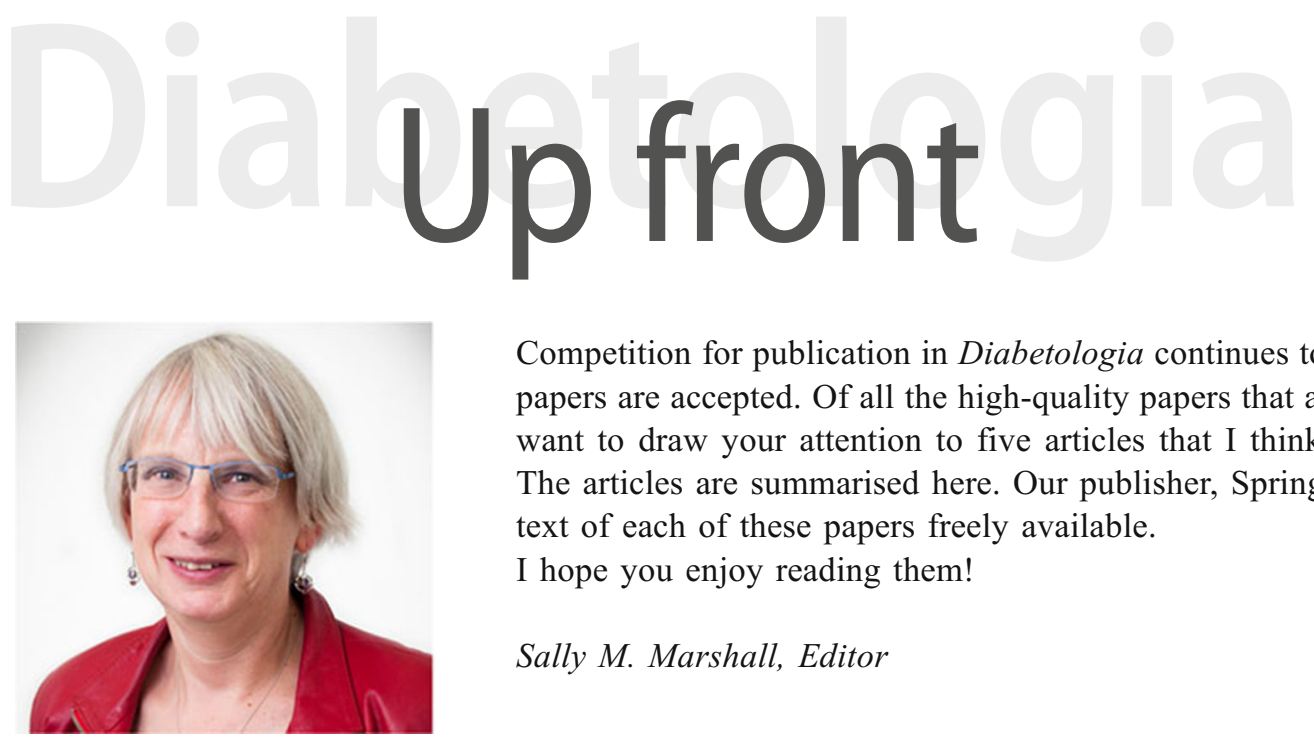

Competition for publication in Diabetologia continues to grow, and less than $20 \%$ of papers are accepted. Of all the high-quality papers that appear in this month's issue I want to draw your attention to five articles that I think are particularly interesting. The articles are summarised here. Our publisher, Springer, has kindly made the full text of each of these papers freely available.

I hope you enjoy reading them!

Sally M. Marshall, Editor

\section{Myokines in metabolic homeostasis and diabetes}

Jürgen Eckel

Physical activity triggers the release of a host of myokines, a group of molecules that integrate contracting muscle into the complex network of organ communication, consisting of metabolites, exosomes and many other crosstalk signals. In this issue, Jürgen Eckel (https://doi.org/10.1007/s00125-019-4927-9) reviews how myokines, as part of the organ crosstalk network, serve to communicate immediate and long-term information for functional adjustments in different tissues, with a significant role in positive metabolic control. In humans, the functional allocation of myokines has remained rather limited. Eckel suggests that future studies are needed to address the specific role of myokines in concert with other crosstalk molecules in humans, and the auto- and paracrine actions of these molecules. This may provide new insight into muscle physiology, as well as indicating myokines with therapeutic potential in diabetes.

(t) The figure from this review is available as a downloadable slide.

\section{Clinical aspects of heart failure in individuals with diabetes}

Christa D. Bowes, Lillian F. Lien, Javed Butler

Cardiovascular outcome (CVO) trials with novel glucoselowering therapies have provided us with a deeper understanding of the risks and benefits of these agents with respect to cardiovascular outcomes. In turn, CVO trials have allowed individualisation of therapy for patients with diabetes. Since individuals with diabetes are at a higher risk of developing heart failure (HF) and, subsequent to HF development, have much worse prognosis, it is important to consider the issues concerning HF risk and management in these individuals. In this issue, Bowes et al (https://doi.org/10.1007/s00125-0194958-2) summarise available data on the impact of various glucose-lowering therapies on the risks of developing HF and their role in the prevention of incident HF. They state that these data will provide clinicians with further insights to help optimise the management of patients with diabetes with and without HF. (D) The figure from this review is available as a downloadable slide.

New insights into the mechanisms of diabetic complications: role of lipids and lipid metabolism

Stephanie Eid, Kelli M. Sas, Steven F. Abcouwer, Eva L. Feldman, Thomas W. Gardner, Subramaniam Pennathur, Patrice E. Fort

The mechanisms driving the onset and progression of diabetic complications have been extensively studied individually, but the commonalities and specificities between them have received very little attention. The review by Eid et al (https:// doi.org/10.1007/s00125-019-4959-1) in this issue specifically discusses omics-based studies of microvascular complications in both the experimental and clinical settings. The authors place a special emphasis on the emerging role of lipids and lipid metabolism as a central mechanism underlying diabetic complications. The omics-based non-targeted analyses of tissues 
from diabetic individuals and preclinical models following interventions have been key in reshaping our understanding of the pathophysiological mechanisms involved in microvascular complications, forming new hypotheses for therapeutic leads.

(L) The figures from this review are available as a downloadable slideset.

\section{Oestrogen receptor $\beta$ mediates the actions of bisphenol-A on ion channel expression in mouse pancreatic beta cells}

Juan Martinez-Pinna, Laura Marroqui, Abdelkrim Hmadcha, Javier Lopez-Beas, Sergi Soriano, Sabrina Villar-Pazos, Paloma Alonso-Magdalena, Reinaldo S. Dos Santos, Ivan Quesada, Franz Martin, Bernat Soria, Jan-Åke Gustafsson, Angel Nadal

Bisphenol-A (BPA) is an endocrine-disrupting chemical (EDC) found in many widely used products. BPA was detected in the urine of $93 \%$ of US citizens and its concentration in serum reaches between 1 and $25 \mathrm{nmol} / \mathrm{l}$. Epidemiological studies have associated EDC exposure with type 2 diabetes in humans. BPA increases insulin levels and release in the presence of stimulatory glucose concentrations. In this issue, Martinez-Pinna, Marroqui et al (https://doi.org/10.1007/ s00125-019-4925-y) report that BPA, at environmentally relevant doses, modulates the expression of more than 50 genes encoding $\mathrm{Na}^{+}$and $\mathrm{K}^{+}$ion channel subunits in mouse beta cells. BPA treatment resulted in decreased $\mathrm{Na}^{+}$and $\mathrm{K}^{+}$ currents in islets, as well as modifying glucose-induced electrical activity. Using beta cells from oestrogen receptor $\beta$ $(E r \beta)$ knockout mice, the authors demonstrated that the BPA-induced effects in pancreatic islets were dependent on oestrogen receptor $\beta$. They suggest that these results help to explain how BPA regulates insulin content and release and shed light upon the mechanisms by which EDCs with oestrogenic activity exert their diabetogenic activity.

\section{Targeting gut microbiota and barrier function with prebiotics to alleviate autoimmune manifestations in NOD mice}

Camilla H. F. Hansen, Christian S. Larsen, Henriette O. Petersson, Line F. Zachariassen, Andreas Vegge, Charlotte Lauridsen, Witold Kot, Lukasz Krych, Dennis S. Nielsen, Axel K. Hansen

The influence of the intestinal environment on autoimmunity has become very relevant of late. As such, identifying means to target specific microbes and improve intestinal function would be a valuable achievement for future prevention/ treatment strategies for dysbiosis. In this issue, Hansen et al (https://doi.org/10.1007/s00125-019-4910-5) present results that: (1) demonstrate how prebiotic xylooligosaccharides (XOS) can delay diabetes onset and reduce autoimmune reactions towards the pancreatic beta cells and salivary glands in NOD mice; and (2) show that the protective effects offered by XOS were regulated by both microbe-dependent and -independent pathways. The authors suggest that reduced gut permeability and associated induction of anti-inflammatory mucosal immune responses play a central role in protection against autoimmune destruction of beta cells and salivary glands. Considering that many individuals with autoimmunity have dysfunctional barrier function, these findings strongly encourage further testing of the beneficial effects of prebiotics in individuals predisposed to autoimmune diseases, such as type 1 diabetes.

Publisher's Note Springer Nature remains neutral with regard to jurisdictional claims in published maps and institutional affiliations.

All text supplied by the authors. 